pesca degli USA, praticata in acqua dolce, è estratto dai Grandi Laghi. La partecipazione del Canadà è notevolmente inferiore. Circa $35-40 \%$ del prodotto globale deriva dal Lago Erie, $20-30 \%$ dal Lago Michigan e più del $20 \%$ dal Lago Superiore. Nel corso degli anni, l'ammontare spettante alle diverse specie di pesci ha subito notevoli fluttuazioni. Una notevole diminuzione della pesca delle trote, verificata in questi ultimi anni, è dovuta senza dubbio all'immigrazione della lempreda, che proveniente dal Lago Ontario attraverso il Welland Canal ha invaso tutti i laghi.

\title{
EINDRÜCKE EINES FLUGES ÜBER NEPAL
}

Mit 5 Abbildungen

E.Mil Raucit

Ende 1952 war es dem Verfasser zusammen mit vier Experten * der UN vergönnt, einen Orientierungsflug über Nepal zu unternehmen, der einen Überblick über dessen wesentliche Landschaften und deren Probleme gab. In der folgenden Skizze ist versucht, die Ergebnisse des Fluges kurz zusammen zu fassen.

Dic Nalurlandschof. Zunächst vermittelte der Flug einen Einblick in die Topographie des Landes, das trotz seines im ganzen ausgesprochenen Hochgebirgscharakters eine klare Großsgliederung zeigt. Der an sich schon breiter entwickelte $W$ weist zwischen den unproduktiven Hochgebirgszonen im $\mathrm{N}$ und dem ausgesprochenen Waldgürtel der Siwalik und Mahabharat Ranges im S eine breitere Vorgebirgszone auf als der E. Dieses Hochland mit seinen weiten, fruchtbaren Tälern ist dicht bevölkert. Es ermöglicht intensivere Agrarproduktion. Im ganzen zeichnen sich von $\mathbf{N}$ nach S vier Zonen ab, die eigentliche Himalayakette, das Hochland des Vorhimalaya, die davon ganz verschiedenen Züge des Mahabharat- und Siwalik-Gebirge und die vorgelagerte Tiefebene des Tarai (Kartenskizze).

Dem Vegetationsbild entsprechend muß die Niederschlagsmenge ron $E$ nach $W$ stark abnehmen. Das zeigt sich besonders im Tarai, wo die Provinz Morang (im E) von der natürlichen Wasserversorgung profitiert, in der Provinz Saptari das Wasser mit größern Kanälen verteilt und in der Provinz Mahotari gespeichert werden muß. Je weiter wir nach W kommen desto stärker macht sich der Charakter einer Trockenlandschaft bemerkbar. Selbst in der Bergzone ist das veränderte Waldbild für den Beobachter vom Flugzeug aus ein deutliches Zeichen für den zunehmenden Mangel an Wasser für die Vegetation. Auch die Flußläufe scheinen weniger zahlreich und bedeutend zu sein.

Neben den Verwitterungsböden auf anstehendem Gestein nehmen die Aufschwemmungsböden in Nepal einen breiten Raum ein. Aber das Flugbild zeigt, daß jede dieser Bodenarten Flächen von ganz verschiedenen Nutzungswerten aufweist. Neben den produktiven feinerdigen Schwemmlandböden vieler Täler und des vom Bergfuß entfernteren Tara sehen wir größere Gebiete mit Ablagerungen von Sand und Geröllmassen, die sicher der Vegetation nur langsam erschließen und daher vor allem forstwirtschaftlich genützt werden. Der Geologe zeigt uns, daß diese kargen Böden aus der Vervitterung der Siwalik-Kette, die selbst aus Sekundär-Gesteinen besteht, entstanden sind. Aus den Himalaya-Ketten und aus dem Mahabharat-Gebirge kommen feinere Ablagerungen, welche die

* Mit den Geologen Dr. HaGex (Schweiz), dem Forstingenieur RobBe (Frankreich, dem Mol: experten Schul.thess (Schweiz) und dem Pflanzenbauexperten KNursos (Schweden).

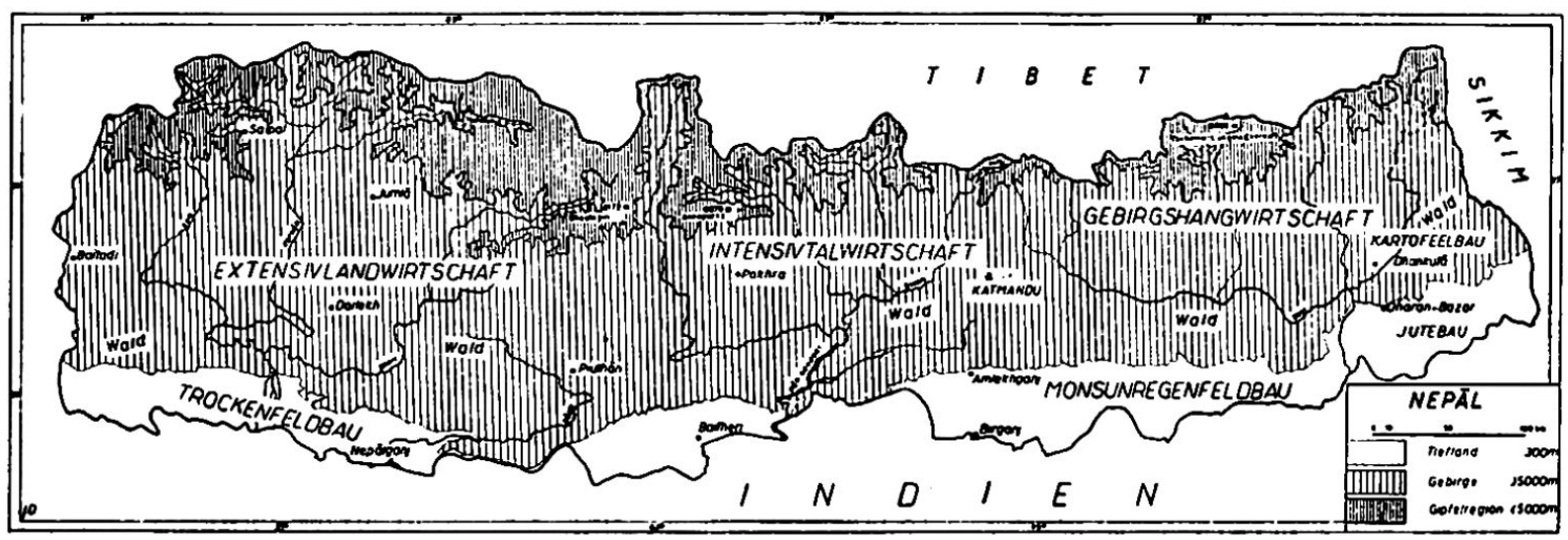

Skizze der Höhen- und Wirtschaftszonen von Nepal 


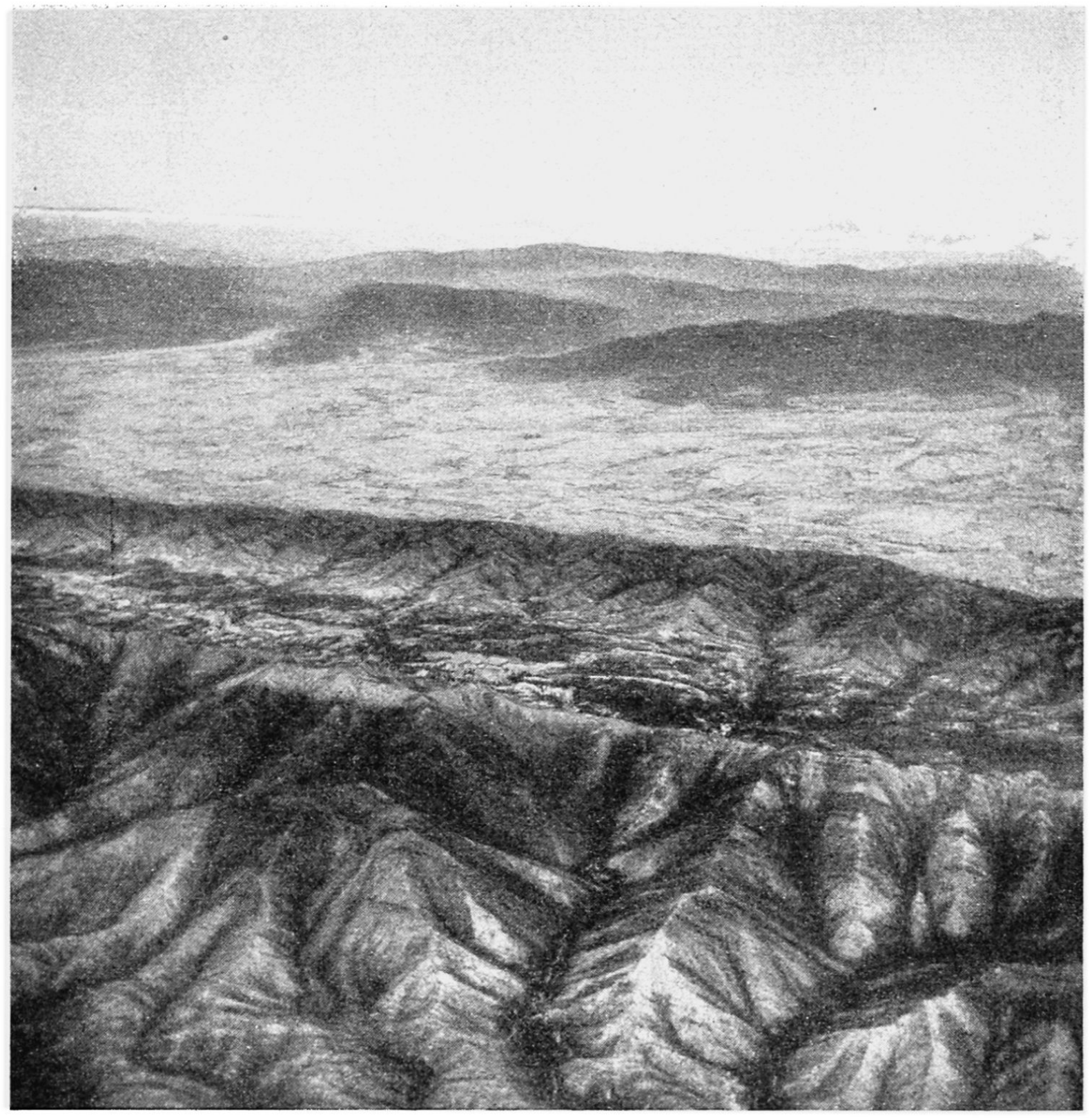

Blick vom Tarai über die Churia Range (Siwalik) und den Oberlauf des Babei Kholas in die Ebene von Bijauri. Dahinter die Mahabharat-Ketten, die Hochflächen von Palpa und die Gipfel der Himalayas (rechts wahrscheinlich der Daulagiri). Photo E. Ravcu

für die Landwirtschaft so wertvollen, tiefgründigen Ebenen bilden. Daß die Mahabharat- wie die Siwalik-Ranges stark bewaldet sind, darf uns nicht täuschen. Der Boden ist bei den ersteren gut. Er entstammt Kalksteinformationen, die einen warmen, tätigen Boden bilden. Die steilen Hänge und die starke Erosion dieser Zone führen zum Waldbau. Soweit es Ebenen und Hochplateaus er lauben, wird Landwirtschaft getrieben. So lassen sich für die landwirtschaftliche Produktion 4 Bodengruppen unterscheiden: Je ein fruchtbarer Verwitterungs- und Aufschwemmungsboden und je ein für die landwirtschaftliche Nutzung ungeeigneter Verwitterungs- und Aufschwemmungsboden. Lokale Ausnahmen ädern nichts an der Regel dieser Gruppierung.

Wirtschaftszonen. Die Hochgebirgszone mit dem schneebedeckten Dach der Welt reicht im $\mathrm{N}$ von $\mathrm{E}$ nach $\mathrm{W}$. Es sind mehrere gegeneinander versetzte, meist über $4000 \mathrm{~m}$ hohe Bergrücken, zwischen denen Pässe und Durchgänge den Verkehr mit den tibetanischen Hochbecken ermöglichen. Auch die Waldzone, die die Siwalikund Mahabharat-Hügelketten bedeckt, ist von $\mathrm{E}$ nach $\mathrm{W}$ durchgehend. Sie wird nach $S$ ins Tarai durch den Niederungswald, der die breiten Schuttkegel bedeckt, zungenartig erweitert; nach $\mathrm{N}$ hin hat der Wald dagegen vor allem die steileren Berghänge zu schützen, ohne die Priorität zu haben. Im Tarai bieten die tiefgrün- 


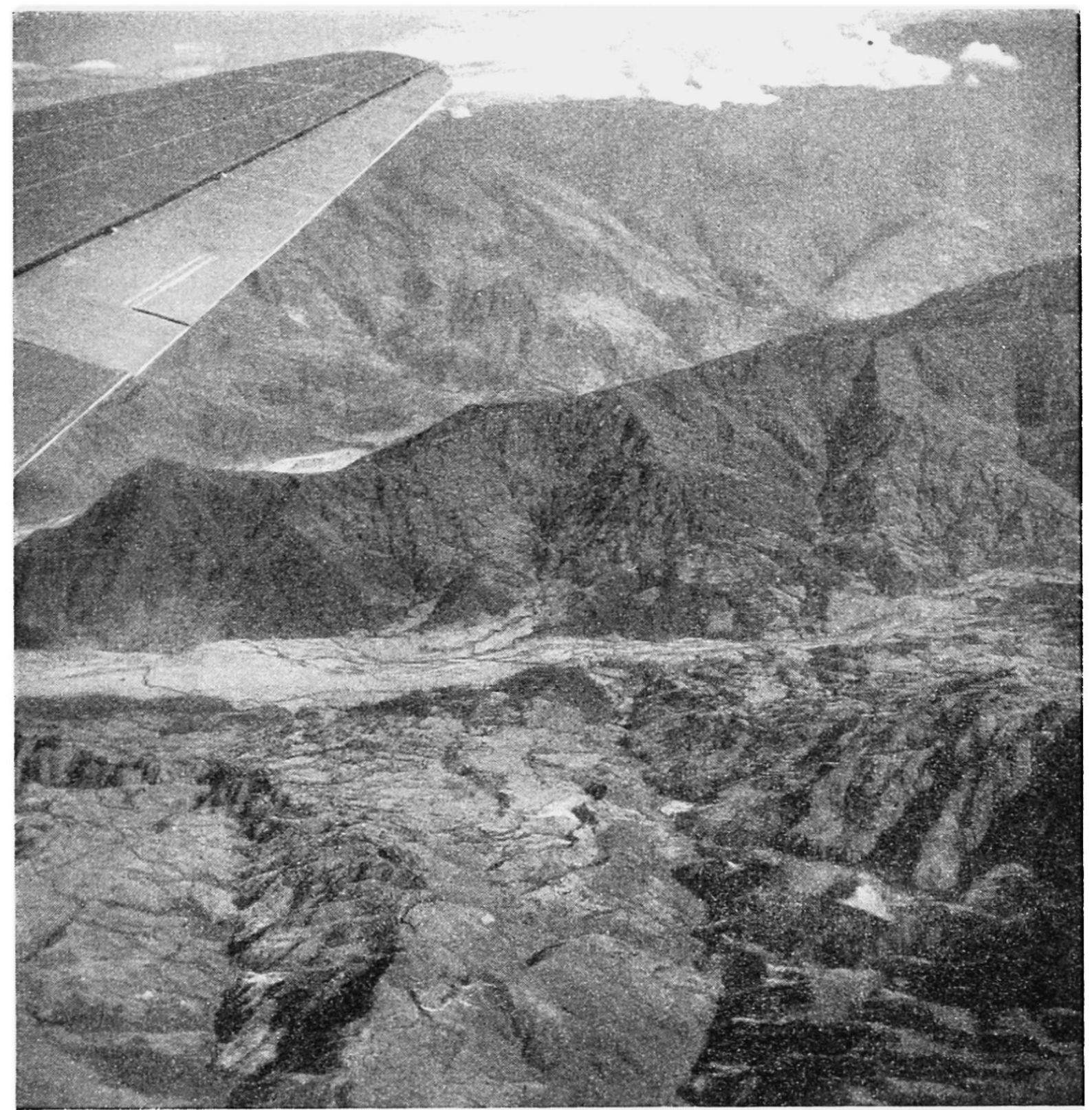

Zwischen Kathmandu und Pokhara. Breite fruchtbare Hänge, bewässerbare Talböden geben einer gesunden Besiedlung Raum. Im Vordergrund der Tadi Khola, hinter dem Gebirgszug der Trisuli. Photo E. Ravch

digen Ablagerungsböden den Kaum für die fruchtbarste entwicklungsfähige Provinz Morang, die Kornkammer von Nepal. Aber auch die sich westwärts anschließende Tiefebene kann bei richtiger Ausnützung des Wassers ein Produktivgebiet werden. Während das Agrargebiet des Tarais dem größeren Produktionsbetrieb mit einer dünneren Bevölkerung vorbehalten bleibt, muß die im Vorgebirge, also zwischen der Hochgebirgs- und Waldzone liegende Agrarlandschaft dicht besiedelt im Kleinbetrieb genutzt werden. Sie wird zwischen Pokhara und Kathmandu zum zweiten wichtigen Agrargebiet Nepals. Die vielen breiten, fruchtbaren und darum dicht besiedelten T̈̈̈ler, die zwischen dem Trisuli und dem Kali Gandaki liegen und von vielen Nebenflüssen bewässert sind, stellen neben der Provinz Morang und dem Kathmandutal wahrscheinlich die wertvollste Agrarzone dar. Es ist falsch, von der Bedeutung von Pokhara zu sprechen; das ganze Gebiet muß und kann gemeinschaftlich entwickelt werden. Der Anschluß dieses Gebietes an Kathmandu würde wesentlich zur Entwicklung des Landes beitragen. 


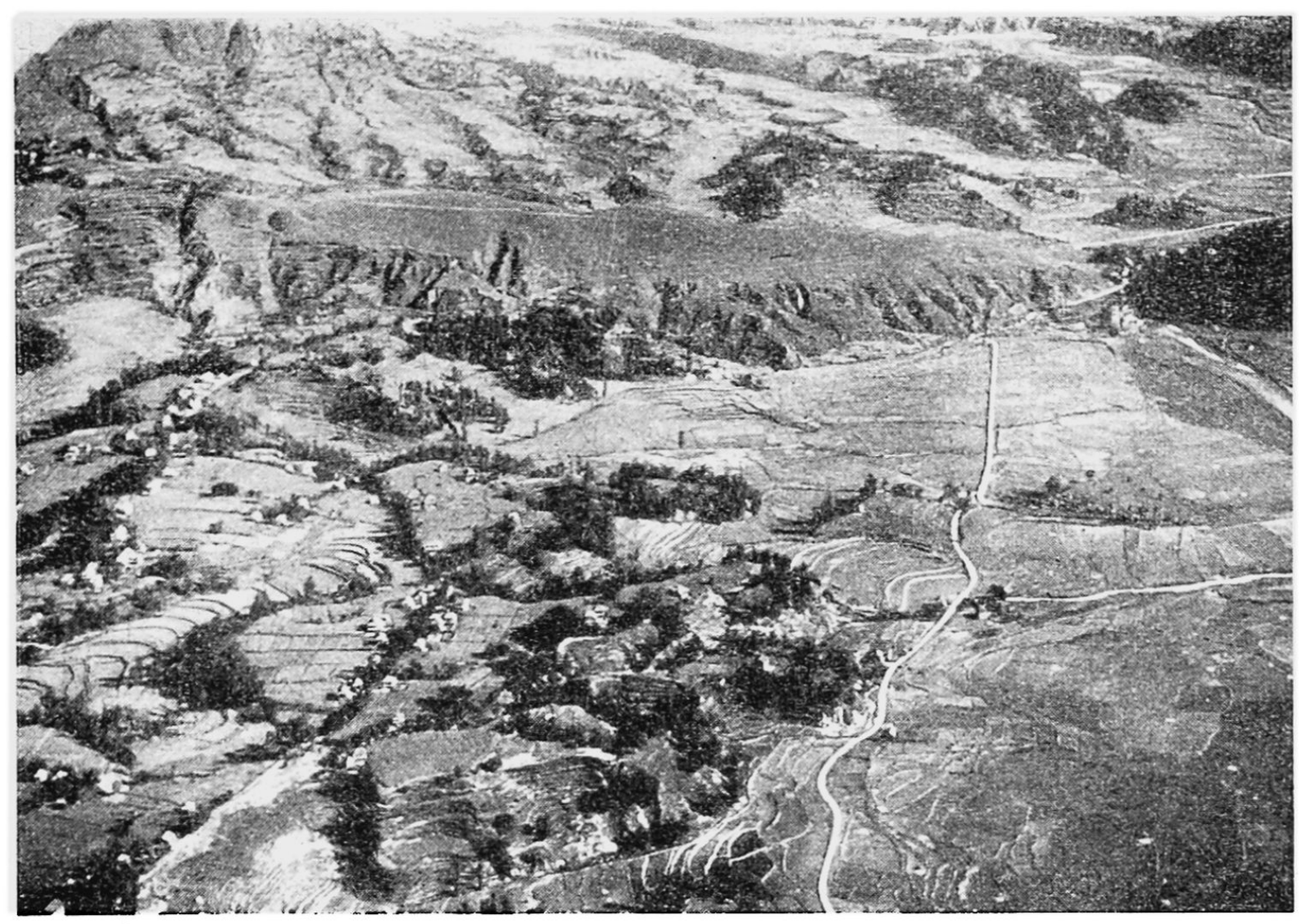

Am Rande des Kathmandutales. Neben den stark terrassierten Aufschwemmungsböden des alten Seebeckens sind die ins Tal einspringenden Riegel der älteren Formationen sichtbar (mit Gras bewachsen). Man sieht die lockere Siedlung. Straße nach Sundarijal. Photo E. RAuch

Dem E fehlen die breiten 'Täler. Hier, wo die Vegetation der günstigeren Niederschläge wegen eine üppigere ist, kann sich der Ackerbau nicht stärker ausbreiten. Erst in den höheren Lagen, und auch da nur mit perennierenden Pflanzen (Graswirtschaft), gewinnt die landwirtschaftliche Produktion Bedeutung. Wir wissen, daß der für den Transport günstiger gelegene Teil des E, also die Höhen um Dhankuta, als Kartoffel- und Cardamumexportgebiet einige Bedeutung haben, aber wir können vom Flugzeug aus kein zusammenhängendes Agrargebiet erkennen. Westlich des Kali Gandaki macht sich der Einfluß des Trockenklimas mehr und mehr bemerkbar. Die Böden scheinen nicht schlecht; trotzdem wird die landwirtschaftliche Nutzung extensiver, die Besiedlung schwächer. Man wird zeitweise an den Jura erinnert. Der Geologe bestätigt die Kalkformation und der Forstexperte schlägt bestockte Weiden vor. Hier mag die Viehhaltung wieder mehr Bedeutung haben, sie wird sich aber nicht so stark entwickeln können wie im E. Eine zwar gesunde Zone, die jedoch nur langsam zu entwickeln ist und die nie die Bedeutung haben wird wie der $\mathrm{E}$.

Folgerungen. Zwei wichtige Ackerbaugebiete stehen im Vordergrund des Interesses: das produktive im West-Tarai (Provinzen Morang, Saptari, Mahotari) und das bevölkerte, leistungsfähige Vorgebirggsgelände zwischen Pokhara und Kathmandu-Valley. Der nördliche $\mathrm{E}$ sollte für die Milchwirtschaft die besten Voraussetzungen haben. Hier ist die Arbeit des Molkereiexperten zu konzentrieren. Entsprechende Ausnahmen vorbehalten, ist das Gebiet der den Himalayas vorgelagerten Bergketten, also der Mahabarat- und namentlich der Siwalik-Zone mit den Schuttkegeln der letzteren, dem Wald vorzubehalten. Das Gesamtbild bestätigt meine letztjährigen Beobachtungen im Raptivalley. Es gilt auch für den breiten Gürtel des innern Tarais und der anschließenden Bergketten. 


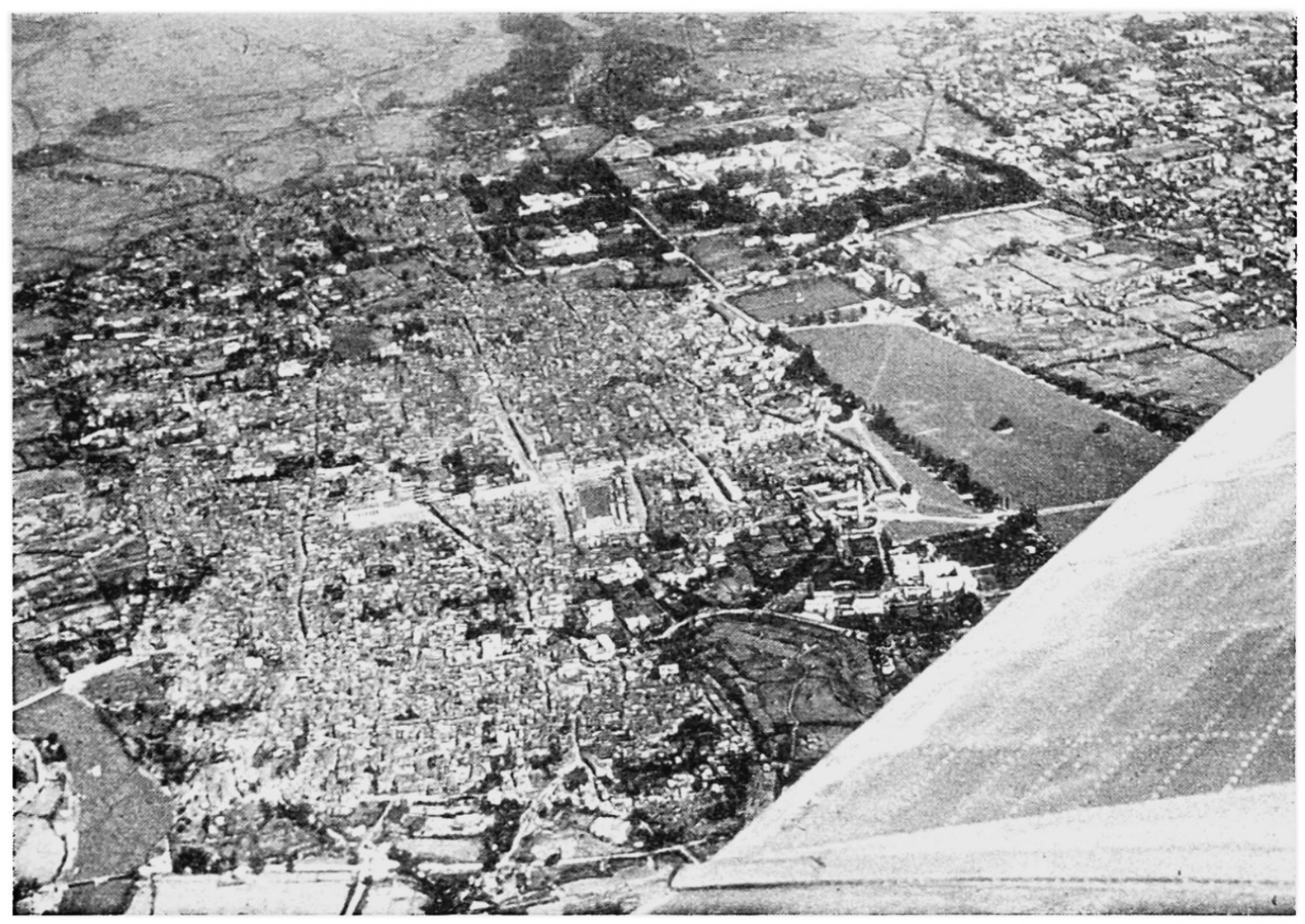

Blick auf Kathmandu. (ian\% links der Vishnumati (Nebenfluß des Bagmatis) rechts das Rechteck der Parad Ground. Hinter demselben rechts der Straße der Königspalast, links der Straße zwei Rana Paläste, dahinter im Park die Englische Botschaft. Yor dem Flügel ein Ranapalast der minaretartige Turm Bhim Sen. Mitte Bild Bank und Lagerhaus. Dahinter das moderne Straßenkreuz mit Kino in der Ecke. In der Verlängerung nach links weißes Gebäude: Alter Regierungspalast, dient offiziellen Empfängen. Der Palast des letzten Maharajas, jetzt Regierungsgebäude, ist rechts des Parad Grounds vom Flügel verdeckt. Außer dem dicht besiedelten Stadtkern im Bilde schließen sich locker bebaute Vororte nach allen Seiten an. Photo li. Raucu

Der Flug hat erneut gezeigt, daß das wichtigste Problem die Verbesserung des Verkehrs darstellt. Zwei Straßen sind vordringlich: die von Pokhara nach Kathmandu und der Ausbau der Verbindung Jogbani-Dharanbazar. Beide sind einfacher und billiger zu erstellen, als allgemein angenommen wird. Das Flugbild bekräftigt frühere Vorschläge, daß die Straße nach W von Kathmandu aus dem Mahesh Khola entlang hinunter bis zum Trisuli und weiter zum Marșandi geführt werden muß. Diese Hauptverkehrsader ermöglicht die Erschließung der Provinzen West I, II, III, IV. Die Straße ist aber auch nötig, um später Mid West Nepal und Far West Nepal mit der Hauptstadt zu verbinden. Eine so aufgelockert besiedelte Landschaft wie West I bis IV kann weder mit Flugzeug noch Bahnen oder durch Kulis erschlossen werden, sondern muß mit einigen Hauptstraßenzügen den Ferntransport mit Kraftwagen ermöglichen. Der Ausbau der Anschlußstraße wird sich den Bedürfnissen entsprechend etappenweise und vielleicht durch die regionalen Organisationen (Local Self Government) durchführen lassen.

Auch bezüglich der Bewässerung bestätigt der Flug, daß große und fruchtbare Gebiete mit relativ einfachen und billigen Mitteln in ihrer Produktion gesteigert werden können. 'Weder im 'Tarai noch im Gebirge sind große Dämme und lange Wasserzufuhren notwendig, sondern der Ausbau von vielen kleinen lokalen Gemeinschaftssystemen führt am besten und billigsten zur Entwicklung des Landes. 
Das Flugbild hat wohl allen Teilnehmern neben dem großen Genuß wertvolle Hinweise und Kenntnisse verschafft, die die weitere Arbeit fördern werden. Aber darüber waren sich alle einig: dieser Übersicht muß die Feinarbeit, die Vertiefung des Flugbildes, die Aussonderung von Spezial- und Lokalbedingungen, der Kontakt mit den Menschen und nicht zuletzt die praktische Förderung des Fortschrittes folgen. Wir danken der Regierung Seiner Majestät des Königs für die Organisation des Fluges und hoffen, daß unsere Arbeiten dem Fortschritt des Landes dienen werden.

\section{VOL SUR LE NEPAL}

Vu de l'avion, le Népal présente une configuration de paysages bien distincts. Par rapport à la topographie, il est possible de diviser le territoire népalais en 4 parties: la zone improductive de la haute montagnè, la zone forestière des contreforts formés par les chaines du Mahabharat et du Sivalik, le plateau de population relativement dense, situé entre les deux premières zones et enfin, en bordure, la plaine qui fait partie de la vallée du Gange (Taraï). La végétation indique une forte diminution de la quantité des précipitations de l'est à l'ouest. Les sols désagrégés des Himalayas semblent infiniment plus féconds que ceux de la chaine du Sivalik. Les terrains sédimentaires ont, eux aussi, différents degrés de fertilité. Ceux-ci dépendent de leur origine. Ainsi donc on parvient à distinguer des zones économiques différentes. A l'est, la plaine méridionale avec sa province de Morang, productrice de jute, et les larges vallées s'étendant entre Kathmandu et Pokhara sont les contrées agricoles de grande valeur économique. Sur les pentes plus escarpées de l'est et sur le plateau de la partie ouest qui est plus éloignée, l'économie pastorale domine.

\section{IN VOLO SOPRA IL NEPAL}

Visto dall'alto, il territorio del Nepal può essere suddiviso in diversi paesaggi. Topograficamente si può distinguere: la zona di alta montagna improduttiva; la zona boschiva delle catene prehymalaiane di Mahabarat e di Siwalik; tra mezzo, la zona di altopiano con una densità di popolazione relativamente alta; l:avamposto bassopiano di Tarai che già appartiene alla vallata del Gange. La variazione della vegetazione rispecchia una forte diminuzione delle precipitazioni da $\mathrm{E}$ verso $\mathrm{O}$. I terreni di alterazione dell'Hymalaia sembrano notevolmente migliori di quelli delle catene prehymalaiane di Siwalik. I terreni sedimentari rivelano pure una feracità variabile a seconda della provenienza del materiale alluvionale. Queste diversità fisiche si rispecchiano nelle diverse zone economiche: il bassopiano occidentale di Tarai con la provincia di Morang dedita alla juta e le ampie vallete interposte alle catene prehymalaiane, tra Katmandu e Pokhara, sono i territori agricoli più fertili; sui versanti più ripidi della regione orientale e sul lontano altopiano occidentale, sfruttato con sistema estensivo, domina in modo più marcato l'economia dei pascoli.

\section{DIE DECKENTHEORIE \\ IM GEOGRAPHIE- UND GEOLOGIEUNTERRICHT DER MITTELSCHULE \\ Eugen Hess}

Mit 6 Figuren

\section{ZIELSETZUNG}

Dem Geographielehrer der Mittelschule ist neben andern nicht unmittelbar in sein Fach gehörigen Aufgaben (Bevölkerungs-, Siedlungs-, Völkerkunde usw.) auch die Einführung in die Geologie aufgetragen, wobei — insbesondere in einem Hochgebirgslande wie der Schweiz - naturgemäß die Frage aktuell wird, ob und wie die Deckentheorie Berücksichtigung zu erfahren habe. Dieses Thema soll im folgenden einer Beleuchtung unterzogen werden.

Es liegt im Wesen der Forschung, daß zuweilen Beobachtungen gemacht werden, die nicht in bekannte Rahmen passen; für solche muß eine neue Betrachtungsweise gesucht werden, $d$. $h$. eine neue Theorie, etwa von der Art der Molekular-oder Elektronentheorie. Eine solche Theorie hat 